\title{
Inertia effect analysis of a half-plane with an induced crack under thermal loading using hyperbolic heat conduction
}

\author{
Wei $\mathbf{L i}^{1,2}$, Jia Li $^{1, *}$, Radhi Abdelmoula ${ }^{1}$, Fan Song $^{2}$, and Chi-Ping Jiang ${ }^{2,3}$ \\ ${ }^{1}$ LSPM, CNRS UPR 3407 University Paris XIII, 99 Avenue Jean-Baptiste Clement, Villetaneuse, 93430, France \\ ${ }^{2}$ State Key Laboratory of Nonlinear Mechanics (LNM), Institute of Mechanics, Chinese Academy of Sciences, Beijing \\ 100190, China \\ ${ }^{3}$ Beijing University of Aeronautics \& Astronautics, Beijing, 100191, China
}

Received 12 May 2015, revised 21 July 2015, accepted 11 October 2015

Published online 24 November 2015

Key words Thermal stress, inertia effect, hyperbolic heat conduction, Fourier transform, singular integral equations.

This paper considers the crack problem for a semi-infinite plate subjected to a thermal shock by using hyperbolic heat conduction equations and equations of motion. The Laplace transform and Fourier transform are used to reduce the problem to a system of singular integral equations which are solved numerically. Numerical results are presented illustrating the influence of non-Fourier effect and inertia effect on the stress intensity factor and normalized crack opening displacements. The results show that the stress intensity factors have higher amplitude and an oscillating feature comparing to those obtained under the conventional Fourier thermal conduction condition and quasi-static hypothesis. These results can help understand the crack behaviors of advanced materials under thermal impact loading.

(C) 2015 WILEY-VCH Verlag GmbH \& Co. KGaA, Weinheim

\section{Introduction}

Engineering thermo-mechanical structure components can be subjected to severe thermal loading such as extremely powerful laser impact or rapid quenching with liquids from high temperature. Unconventional phenomena can be observed and may play an important role in damage and fracture of the materials. The sources of these phenomena include the non-Fourier heat conduction effect, the inertia effect etc. It was reported that in such situations the Fourier heat conduction model becomes inaccurate [1]. Also, the inertia forces are not negligible and important stress waves can be induced [2]. These phenomena are particularly pronounced in the cases of the very small length and time scales $[3,4]$. The thermal stresses induced by these effects need to be evaluated accurately, especially around micro-cracks, cavities or other defects.

Moreover, the existence of the cracks can strongly influence the heat conductivity for some materials [5]. The interaction of all these factors makes the accurate stress analysis extremely complicated.

In this work, we focus our attention on stress analysis in a cracked body under a thermal load by considering both the non-Fourier effect and inertia forces.

Studies of the thermal stress field distribution in a cracked elastic body have been performed since 1950s [6-9]. In recent years, increasing investigations have been devoted to solve the crack problems under thermal loading in advanced materials [10-17]. However, in most of the models solving the temperature fields and stress fields, non-Fourier effect and the inertia effect are often be neglected due to their complexity. In the thermal shock experiment of Shao [18], they find the cracks parallel to the heating surfaces, which cannot be explained by the quasi-static thermoelastic theory. In order to make the results more accurate, Chen [19-21] developed the theory by using hyperbolic heat conduction equations instead of parabolic heat conduction equations. Within the framework of non-classical heat conduction, Fu [22] obtained the general solutions for the cylinder model with an edge circumferential crack under thermal loading; Wang [23] studies the fracture behavior of a thermoelastic cylinder subjected to a sudden temperature change on its outer surface. On the basis of the found temperature field, the Airy function technique was applied to evaluate the stress concentration around cracks.

However, if we introduce the inertia effect to the stress analysis, the equations of motion have to be used in the solution of the problem. The Airy function method is no longer applicable in this case. New solution techniques should be developed.

In this article, we analyze the transient thermal stresses around a crack in a semi-infinite, thermo-elastic body under a thermal impact using the hyperbolic heat conduction and the wave equation. The crack lies parallel to the free surface under plane elasticity conditions and the crack faces are assumed to be perfectly thermal insulated. We use uncoupled thermo-elastic theory which has been proved not producing significant errors in most of the usual engineering applications

* Corresponding author E-mail: jia.li@univ-paris13.fr 
$[24,25]$. Fourier transforms are employed to derive the singular integral equations about the temperature and the thermal stress field. The singular integral equations are solved numerically and the asymptotic fields around the crack tips are obtained. Laplace inversion is used to get the temperature and stress fields in the time domain. The results of the dynamic stress intensity factors and normalized crack opening displacements plotted versus dimensionless time and dimensionless coordinates.

\section{Statement of the problem and basic equations}

We consider a thin half-infinite plate under transient thermal loading which contains a straight crack of length $2 r$ parallel to the free surface, as shown in Fig. 1. A Cartesian coordinate system is established with the $x$-axis coincided with the crack plane and the origin at the crack center. We assume that the material is homogeneous, isotropic and linearly elastic. In this work, only thermal loading is considered in evaluating the temperature and stress fields. The initial temperature is assumed to be uniform and equals to $T_{0}$ in the plate. Then the plate is suddenly heated to a fixed temperature $T_{\infty}$ at its free surface, $y=-r l$, where $l$ is a strictly positive real. The crack surface is assumed to be thermally insulated. Additionally, the thermal-elastic coupling is neglected in this study.

In order to assess the influence of the non-Fourier and the dynamic effect of the thermal shock, we adopt the hyperbolic heat conduction equations in temperature field evaluation and introduce the inertia effect in stress field to the equilibrium equations.

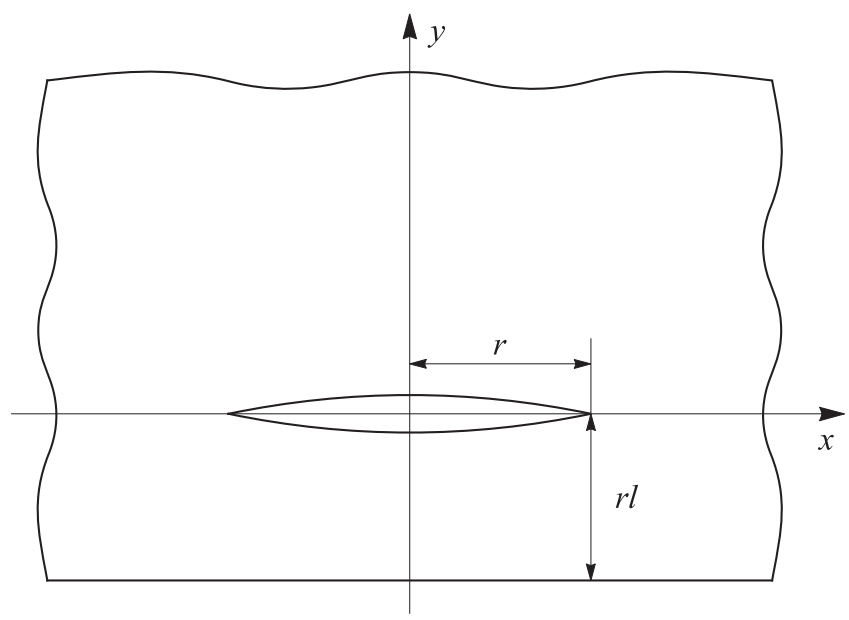

Fig. 1 Model of semi-infinite medium.

In the following, we present the detailed mathematical formulation for the resolution of the above-defined thermal shock problem.

\subsection{Heat conductions}

The famous Fourier's law of heat conduction presents a linear relationship between the heat flux $(\vec{q})$ through a material and the gradient of temperature (T), whose differential form is $\vec{q}=-k \nabla T$, where $k$ is the material thermal conductivity and $\nabla$ is the gradient operator. In principle, however, the Fourier's law leads to an unphysical infinite heat propagation speed within a continuum for transient heat conduction processes because of its parabolic characteristics. To overcome this contradiction, a hyperbolic model with a time $(t)$ dependent term has been proposed, which is named as the CattaneoVernotte model $[26,27]$. The introduced time-derivative term describes a wave nature of heat propagation at a finite speed, which has been proved in both theory and experiments [27-29]. The hyperbolic heat conduction equation for isotropic bodies writes

$$
\vec{q}+\tau_{0} \frac{\partial \vec{q}}{\partial t}=-k \nabla T
$$

where $\tau_{0}$ is the thermal relaxation time. 
For the rigid stationary material without internal heat generation, energy balance within a control mass can be expressed as

$$
\rho C_{p} \frac{\partial T}{\partial t}=-\nabla \vec{q}
$$

where $\rho$ and $C_{p}$ are the mass density and the specific heat capacity, respectively.

Incorporating Eq. (1) in Eq. (2) leads to the following hyperbolic heat conduction equation,

$$
a \cdot \Delta T=\frac{\partial T}{\partial t}+\tau_{0} \frac{\partial^{2} T}{\partial t^{2}}
$$

where $a=\frac{k}{\rho C_{p}}$ stands for thermal diffusivity and $\Delta$ is the Laplace differential operator, respectively.

By introducing the following dimensionless coordinates, time and temperature

$$
(X, Y)=\frac{(x, y)}{r}, \quad \tau=\frac{a t}{r^{2}}, \quad \theta=\frac{T-T_{0}}{T_{\infty}-T_{0}}
$$

where $\mathrm{T}_{0}$ is the initial temperature of the plate and the governing Eq. (3) has the following dimensionless forms

$$
\nabla^{2} \theta=\frac{\partial \theta}{\partial \tau}+\tau_{1} \frac{\partial^{2} \theta}{\partial \tau^{2}}
$$

where

$$
\tau_{1}=\frac{a \tau_{0}}{r^{2}} .
$$

Here the gradient operator $\nabla$ is related to the dimensionless coordinates $X$ and $Y$.

The hyperbolic heat Eq. (5) is subjected to the following boundary and initial conditions written in dimensionless forms:

$$
\left\{\begin{array}{l}
(a): \theta(Y=-l)=1 \\
(b):\left.\frac{\partial \theta}{\partial Y}\right|_{Y \rightarrow 0^{-}}=\left.\frac{\partial \theta}{\partial Y}\right|_{Y \rightarrow 0^{+}}=0 \quad|X| \leq 1 \\
(c):\left.\frac{\partial \theta}{\partial Y}\right|_{Y \rightarrow 0^{-}}=\left.\frac{\partial \theta}{\partial Y}\right|_{Y \rightarrow 0^{+}} \\
(d):\left.\theta\right|_{Y \rightarrow 0^{-}}=\left.\theta\right|_{Y \rightarrow 0^{+}} \\
(e):\left.\theta\right|_{\tau=0}=0 \\
(f):\left.\frac{\partial \theta}{\partial \tau}\right|_{\tau=0}=0
\end{array}|X|>1\right.
$$

\subsection{Thermal-elastic field equations}

In the following, we focus on attention in resolving the plane-stress thermal-elastic problem under the hypothesis

$$
\sigma_{z z}=\tau_{x z}=\tau_{y z}=0 .
$$

The basic equations of plane thermal stress problems for nonhomogeneous isotropic elastic body are:

1. Equations of motion including the inertia effect:

$$
\sigma_{i j, j}=\rho \ddot{u}
$$

2. Strain-displacement relations:

$$
\varepsilon_{i j}=\frac{1}{2}\left(u_{i, j}+u_{j, i}\right)
$$


3. Thermal-elastic stress-strain relations:

$$
\left\{\begin{aligned}
\sigma_{x x} & =\frac{E}{1-v^{2}}\left[\varepsilon_{x x}+v \varepsilon_{y y}-(1+v) \alpha\left(T-T_{0}\right)\right] \\
\sigma_{y y} & =\frac{E}{1-v^{2}}\left[v \varepsilon_{x x}+\varepsilon_{y y}-(1+v) \alpha\left(T-T_{0}\right)\right] \\
\sigma_{x y} & =\frac{E}{1+v} \varepsilon_{x y}
\end{aligned}\right.
$$

where $E, v$, and $\alpha$ are the Young's modulus, the Poisson's ratio, and the coefficient of linear thermal expansion, respectively. The index $i, j$ refer to the coordinate $x, y$. It is to notice that in this paper, the italic letter " $i$ " is used as a subscript. It should not be confounded with the regular script "i", which stands for the imaginary unit.

Besides (4), we introduce some other dimensionless quantities:

$$
\sigma_{I J}=\frac{(1-v) \sigma_{i j}}{E \alpha\left(T_{\infty}-T_{0}\right)},(u, v)=\frac{\left(u_{x}, u_{y}\right)}{r(1+v) \alpha\left(T_{\infty}-T_{0}\right)}
$$

where the index $I, J$ refer to the coordinate $X, Y$.

By introducing the dimensionless variables defined in (12) into (10) and (11), we obtain stress-strain relationships in dimensionless form:

$$
\left\{\begin{array}{l}
\sigma_{X X}=\frac{\partial u}{\partial X}+v \frac{\partial v}{\partial Y}-\theta \\
\sigma_{Y Y}=v \frac{\partial u}{\partial X}+\frac{\partial v}{\partial Y}-\theta \\
\tau_{X Y}=\frac{1-v}{2}\left(\frac{\partial u}{\partial Y}+\frac{\partial v}{\partial X}\right)
\end{array}\right.
$$

The equations of motion become:

$$
\left\{\begin{array}{c}
\frac{\partial \sigma_{X X}}{\partial X}+\frac{\partial \tau_{X Y}}{\partial Y}=\tau_{2}{ }^{2} \frac{(1-v)^{2}}{1-2 \nu} \frac{\partial^{2} u}{\partial \tau^{2}} \\
\frac{\partial \tau_{X Y}}{\partial X}+\frac{\partial \sigma_{Y Y}}{\partial Y}=\tau_{2}{ }^{2} \frac{(1-v)^{2}}{1-2 \nu} \frac{\partial^{2} v}{\partial \tau^{2}}
\end{array}\right.
$$

By substituting the equations of motion (14) with Eq. (13), and by using the dimensionless quantities, we obtain:

$$
\left\{\begin{array}{l}
\frac{\partial^{2} u}{\partial X^{2}}+\frac{1+v}{2} \frac{\partial^{2} v}{\partial X \partial Y}+\frac{1-v}{2} \frac{\partial^{2} u}{\partial Y^{2}}-\tau_{2}{ }^{2} \frac{(1-v)^{2}}{1-2 v} \frac{\partial^{2} u}{\partial \tau^{2}}=\frac{\partial \theta}{\partial X} \\
\frac{\partial^{2} v}{\partial Y^{2}}+\frac{1+v}{2} \frac{\partial^{2} u}{\partial X \partial Y}+\frac{1-v}{2} \frac{\partial^{2} v}{\partial X^{2}}-\tau_{2}{ }^{2} \frac{(1-v)^{2}}{1-2 v} \frac{\partial^{2} v}{\partial \tau^{2}}=\frac{\partial \theta}{\partial Y}
\end{array}\right.
$$

where $\tau_{2}=\frac{a}{V r}$ with $V=\sqrt{\frac{E(1-v)}{\rho(1+v)(1-2 v)}}$ being the stress wave velocity.

The dimensionless parameter $\tau_{2}$, which we named "inertia factor", plays an important role in describing the dynamic behavior of a cracked solid under thermal loading. The physical implication of this parameter in stress concentration near the crack tips will be discussed afterward in this paper. 
In the present problem, the mechanical conditions can be expressed as follows:

$$
\left\{\begin{array}{l}
(a): \tau_{X Y}(X,-l)=0 \\
(b): \sigma_{Y Y}(X,-l)=0 \\
(c): \tau_{X Y}(X, \infty)=0 \\
(d): \sigma_{Y Y}(X, \infty)=0 \\
(e): \tau_{X Y}\left(X, 0^{+}\right)=\tau_{X Y}\left(X, 0^{-}\right)=0 \\
(f): \sigma_{Y Y}\left(X, 0^{+}\right)=\sigma_{Y Y}\left(X, 0^{-}\right)=0 \\
(g): \tau_{X Y}\left(X, 0^{+}\right)=\tau_{X Y}\left(X, 0^{-}\right) \\
(h): \sigma_{Y Y}\left(X, 0^{+}\right)=\sigma_{Y Y}\left(X, 0^{-}\right) \\
(i): u\left(X, 0^{-}\right)=u\left(X, 0^{+}\right) \\
(j): v\left(X, 0^{-}\right)=v\left(X, 0^{+}\right)
\end{array}\right\} \quad|X|<1
$$

\section{Resolution of the governing equations}

In the following, we present the resolution procedure of the above-defined thermal-elastic equations. The analyticalnumerical methods used in [30,31] are essentially followed in this work. Globally speaking, the Laplace and the Fourier transform techniques are used in resolving the problem [19,20,32]. Since the inertia effect is introduced in the equilibrium equations, the resolution procedure becomes more complex. Special resolution techniques are developed for this paper.

\subsection{Temperature field}

The temperature field in the cracked plate can be obtained by solving the governing equation (5) under initial and boundary conditions (7). Applying the Laplace transform to (5) and (7) leads to:

$$
\begin{aligned}
& \nabla^{2} \bar{\theta}=\left(p+\tau_{1} p^{2}\right) \bar{\theta} \\
& \left\{\begin{array}{ll}
(a): \bar{\theta}(X,-l)=\frac{1}{p} \\
(b): \bar{\theta}(X, \infty)=0 \\
(c): \frac{\partial \bar{\theta}\left(X, 0^{+}\right)}{\partial Y}=\frac{\partial \bar{\theta}\left(X, 0^{-}\right)}{\partial Y}=0 & |X|<1 \\
(d): \bar{\theta}\left(X, 0^{+}, p\right)=\bar{\theta}\left(X, 0^{-}, p\right) \\
(e):\left.\frac{\partial \bar{\theta}}{\partial Y}\right|_{Y \rightarrow 0^{+}}=\left.\frac{\partial \bar{\theta}}{\partial Y}\right|_{y \rightarrow 0^{-}}
\end{array}\right\} \quad|X|>1
\end{aligned}
$$

where $\bar{\theta}(X, Y, p)=\int_{0}^{\infty} \theta(X, Y, \tau) \mathrm{e}^{-p \tau} d \tau$ is the Laplace transform of dimensionless temperature.

The general solution of (17) can be solved by using Fourier transform method, temperature in Laplace-Fourier space can be written as:

$$
\tilde{\theta}(\xi, Y, p)= \begin{cases}c_{1}(\xi) \mathrm{e}^{-m Y}+c_{2}(\xi) \mathrm{e}^{m Y} & -l<Y<0 \\ c_{3}(\xi) \mathrm{e}^{-m Y}+c_{4}(\xi) \mathrm{e}^{m Y} & Y>0\end{cases}
$$

where $m=\sqrt{\xi^{2}+p+\tau_{1} p^{2}}$ and $\tilde{\bar{\theta}}(\xi, Y, p)=\int_{-\infty}^{\infty} \bar{\theta}(X, Y, p) \mathrm{e}^{\mathrm{i} \xi x} d x, c_{i}(\xi)(i=1,2,3,4)$ are unknown parameters to be determined.

Now we introduce the following density function in Laplace space:

$$
\bar{\phi}(X)=\left.\frac{\partial \bar{\theta}}{\partial X}\right|_{Y \rightarrow 0^{+}}-\left.\frac{\partial \bar{\theta}}{\partial X}\right|_{Y \rightarrow 0^{-}}
$$

It is clear that from the boundary condition $(c)$ in (18), we have:

$$
\int_{-1}^{1} \bar{\phi}(X) d X=0
$$


and

$$
\bar{\phi}(X)=0,|X|>1 .
$$

By applying the boundary conditions (18) and the density function (20), the temperature field in Laplace space can be expressed as:

$$
\bar{\theta}= \begin{cases}\frac{1}{2 \pi} \int_{0}^{\infty} \frac{\mathrm{e}^{-m(2 l+Y)}-\mathrm{e}^{m Y}}{\xi} \tilde{\bar{\phi}} \sin (\xi X) d \xi+\frac{\mathrm{e}^{-m_{0}(l+Y)}}{p}-l<Y \leq 0 \\ \frac{1}{2 \pi} \int_{0}^{\infty} \frac{\left(1+\mathrm{e}^{-2 l m}\right) \mathrm{e}^{-m Y}}{\xi} \tilde{\bar{\phi}} \sin (\xi X) d \xi+\frac{\mathrm{e}^{-m_{0}(l+Y)}}{p} \quad Y>0\end{cases}
$$

where $\tilde{\bar{\phi}}=\int_{-\infty}^{\infty}\left(\int_{0}^{\infty} \phi \mathrm{e}^{-p \tau} d \tau\right) \mathrm{e}^{\mathrm{i} \xi X} d \xi$ is the Fourier transform of Laplace transform of $\phi ; m=\sqrt{\xi^{2}+p+\tau_{1} p^{2}}$ and $m_{0}=\sqrt{p+\tau_{1} p^{2}}$.

Substituting (23) into (c) in (18), we get the singular integral equation for $\phi(X)$ as follows:

$$
\int_{-1}^{1} \bar{\phi}(\eta)\left[\frac{1}{\eta-X}+k(X, \eta)\right] d \eta=\frac{2 \pi m_{0} \mathrm{e}^{-m_{0} l}}{p}
$$

where

$$
k(X, \eta)=\int_{0}^{\infty}\left[1-\frac{m}{\xi}\left(1+\mathrm{e}^{-2 m l}\right)\right] \sin \xi(X-\eta) d \xi
$$

The integral equation (24) under the singles-value condition (21) has the following form of solution [33]:

$$
\bar{\phi}(X)=\frac{\bar{\Phi}(X)}{\sqrt{1-X^{2}}}
$$

where $\bar{\Phi}(X)$ is bounded and continuous on the interval [-1, 1]. Following the numerical techniques proposed by [33], Eqs. (21) and (24) can be solved at discrete points as:

$$
\left\{\begin{array}{l}
\sum_{j=1}^{n} \frac{\pi}{n} \bar{\Phi}\left(\eta_{j}\right)\left[\frac{1}{\eta_{j}-X_{i}}+k\left(X_{i}, \eta_{j}\right)\right]=\frac{2 \pi m_{0} \mathrm{e}^{-m_{0} l}}{p} \\
\sum_{j=1}^{n} \frac{\pi}{n} \bar{\Phi}\left(\eta_{j}\right)=0
\end{array}\right.
$$

where the Gauss-Chebyshev points are defined as

$$
\eta_{j}=\cos \left(\frac{2 j-1}{2 n} \pi\right) \quad j=1,2, \ldots, n
$$

and

$$
X_{i}=\cos \left(\frac{i \pi}{n}\right) \quad i=1,2, \ldots, n-1
$$

Once the function $\bar{\Phi}(X)$ is obtained, the temperature fields in $p$-plane can be calculated by using the Chebyshev quadrature for integration as

$$
\bar{\theta}=\left\{\begin{array}{l}
\sum_{i=1}^{n} \frac{1}{n} \bar{\Phi}\left(\eta_{i}\right) \int_{0}^{\infty} \frac{\mathrm{e}^{-m(2 l+Y)}-\mathrm{e}^{m Y}}{2 \xi} \sin \left[\xi\left(X-\eta_{i}\right)\right] d \xi+\frac{\mathrm{e}^{-m_{0}(l+Y)}}{p} \quad-l<Y \leq 0 \\
\sum_{i=1}^{n} \frac{1}{n} \bar{\Phi}\left(\eta_{i}\right) \int_{0}^{\infty} \frac{\left(1+\mathrm{e}^{-2 l m}\right) \mathrm{e}^{-m Y}}{2 \xi} \sin \left[\xi\left(X-\eta_{i}\right)\right] d \xi+\frac{\mathrm{e}^{-m_{0}(l+Y)}}{p} \quad Y>0
\end{array}\right.
$$

Temperature in time domain can be given by applying the inverse Laplace transform, as detailed in [20]. 


\subsection{Displacement and stress fields}

Once the temperature field in Laplace space is found, it is introduced to the equations of motion (15) to determine the displacement and stress fields. To this end, we first write the equations of motion (15) and the boundary conditions (16) in Laplace space by means of the Laplace transform, then the Fourier transform with respect to $X$ coordinate is applied to these equations such that a non-linear ordinary differential equation system in $Y$-coordinate can be obtained in Fourier-Laplace space. These differential equations are then resolved by using appropriate numerical methods. Inverse Fourier transform then inverse Laplace transform of these solutions provide the displacement and stress fields with which some important measures such as the dynamic stress intensity factors of the crack, the crack opening distance etc. can be obtained.

Firstly, using the Laplace transform to Eqs. (15) and (16), we obtain:

$$
\left\{\begin{array}{l}
\frac{\partial^{2} \bar{u}}{\partial X^{2}}+\frac{1+v}{2} \frac{\partial^{2} \bar{v}}{\partial X \partial Y}+\frac{1-v}{2} \frac{\partial^{2} \bar{u}}{\partial Y^{2}}-\tau_{2}{ }^{2} \frac{(1-v)^{2}}{1-2 v} p^{2} \bar{u}=\frac{\partial \bar{\theta}}{\partial X} \\
\frac{\partial^{2} \bar{v}}{\partial Y^{2}}+\frac{1+v}{2} \frac{\partial^{2} \bar{u}}{\partial X \partial Y}+\frac{1-v}{2} \frac{\partial^{2} \bar{v}}{\partial X^{2}}-\tau_{2}{ }^{2} \frac{(1-v)^{2}}{1-2 v} p^{2} \bar{v}=\frac{\partial \bar{\theta}}{\partial Y}
\end{array}\right.
$$

$$
\left\{\begin{array}{l}
(a): \overline{\tau_{X Y}}(X,-l)=0 \\
(b): \overline{\sigma_{Y Y}}(X,-l)=0 \\
(c): \overline{\tau_{X Y}}(X, \infty)=0 \\
(d): \overline{\sigma_{Y Y}}(X, \infty)=0 \\
(e): \overline{\tau_{X Y}}\left(X, 0^{+}\right)=\overline{\tau_{X Y}}\left(X, 0^{-}\right)=0 \\
(f): \overline{\sigma_{Y Y}}\left(X, 0^{+}\right)=\overline{\sigma_{Y Y}}\left(X, 0^{-}\right)=0 \\
(g): \overline{\tau_{X Y}}\left(X, 0^{+}\right)=\overline{\tau_{X Y}}\left(X, 0^{-}\right) \\
(h): \overline{\sigma_{Y Y}}\left(X, 0^{+}\right)=\overline{\sigma_{Y Y}}\left(X, 0^{-}\right) \\
(i): \bar{u}\left(X, 0^{+}\right)=\bar{u}\left(X, 0^{-}\right) \\
(j): \bar{v}\left(X, 0^{+}\right)=\bar{v}\left(X, 0^{-}\right)
\end{array}\right\} \quad|X| \geq 1
$$

where $(\bar{u}, \bar{v})=\int_{0}^{\infty}(u, v) \mathrm{e}^{-p \tau} d \tau$ and $\overline{\sigma_{I J}}=\int_{0}^{\infty} \sigma_{I J} \mathrm{e}^{-p \tau} d \tau$ are the Laplace transform of the displacement components and stress components, respectively.

Then applying the Fourier transform to (31)-(32), gives

$$
\left\{\begin{array}{l}
\frac{d^{2} \tilde{\bar{u}}}{d Y^{2}}+\beta_{1} \frac{d \tilde{\bar{v}}}{d Y}+\beta_{2} \tilde{\bar{u}}=\beta_{3} \tilde{\bar{\theta}} \\
\frac{d^{2} \tilde{\bar{v}}}{d Y^{2}}+\beta_{4} \frac{d \tilde{\bar{u}}}{d Y}+\beta_{5} \tilde{\bar{v}}=\frac{d \tilde{\bar{\theta}}}{d Y}
\end{array}\right.
$$

$$
\left\{\begin{array}{l}
(a): \tilde{\bar{\tau}}_{X Y}(-l)=0 \\
(b): \tilde{\tilde{\sigma}}_{Y Y}(-l)=0 \\
(c): \tilde{\tilde{\bar{\tau}}}_{X Y}(\infty)=0 \\
(d): \tilde{\bar{\sigma}}_{Y Y}(\infty)=0 \\
(e): F^{-1}\left[\tilde{\tilde{\tau}}_{X Y}(Y)\right]_{Y=0^{+}}=F^{-1}\left[\tilde{\tilde{\tau}}_{X Y}(Y)\right]_{Y=0^{-}}=0 \\
(f): F^{-1}\left[\tilde{\tilde{\sigma}}_{Y Y}(Y)\right]_{Y=0^{+}}=F^{-1}\left[\tilde{\tilde{\sigma}}_{Y Y}(Y)\right]_{Y=0^{-}}=0
\end{array}\right\} \quad|X| \leq 1
$$




$$
\left\{\begin{array}{l}
\tilde{\bar{\sigma}}_{X}=-\mathrm{i} \xi \tilde{\bar{u}}+v \frac{d \tilde{\bar{v}}}{d Y}-\tilde{\bar{\theta}} \\
\tilde{\tilde{\sigma}}_{Y}=-\mathrm{i} \xi v \tilde{\bar{u}}+\frac{d \tilde{\bar{v}}}{d Y}-\tilde{\bar{\theta}} \\
\tilde{\bar{\tau}}_{X Y}=\frac{1-v}{2}\left(\frac{d \tilde{\bar{u}}}{d Y}-\mathrm{i} \xi \tilde{\bar{v}}\right)
\end{array}\right.
$$

where $\tilde{\bar{u}}$ and $\tilde{\bar{v}}$ are the Fourier transform of $\overline{u_{x}}$ and $\overline{u_{y}}$, respectively, and $\beta_{i}(i=1,2,3,4,5)$ are given by:

$$
\left\{\begin{array}{l}
\beta_{1}=-\frac{1+v}{1-v} \mathrm{i} \xi \\
\beta_{2}=-\frac{2}{1-v}\left(\xi^{2}+\tau_{2}^{2} \frac{(1-v)^{2}}{1-2 v} p^{2}\right) \\
\beta_{3}=-\frac{2 \mathrm{i} \xi}{1-v} \\
\beta_{4}=-\frac{1+v}{2} \mathrm{i} \xi \\
\beta_{5}=-\left(\frac{1-v}{2} \xi^{2}+\tau_{2}^{2} \frac{(1-v)^{2}}{1-2 v} p^{2}\right)
\end{array}\right.
$$

By considering the boundary conditions (c), (d) in (34) and the temperature fields, the solution of Eqs. (33), i.e., the displacement field in Laplace-Fourier space can be expressed as:

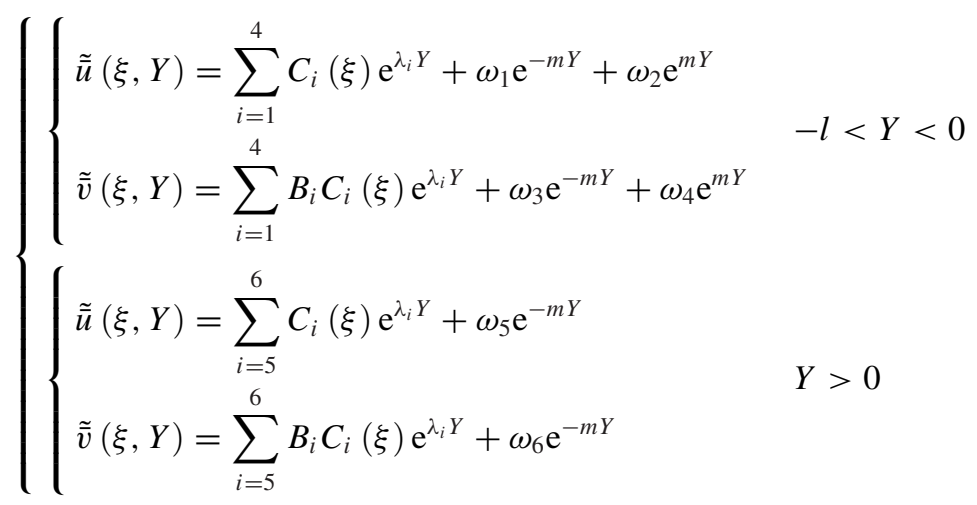

where $C_{i}(\xi)(i=1, \ldots, 6)$ are unknown functions to be determined. Substitute Eq. (37) into (33) result in the following equations:

$$
\left\{\begin{array}{l}
\beta_{1} \lambda_{j} B_{j}+\lambda_{j}{ }^{2}+\beta_{2}=0 \\
\left(\lambda_{j}{ }^{2}+\beta_{5}\right) B_{j}+\beta_{4} \lambda_{j}=0
\end{array}\right.
$$

so $\lambda_{i}(i=1, \ldots, 4)$ can be given by the roots of the following equation:

$$
\lambda^{4}+\left(\beta_{2}+\beta_{5}-\beta_{1} \beta_{4}\right) \lambda^{2}+\beta_{2} \beta_{5}=0
$$

and the coefficients $\lambda_{j}(j=5,6)$ are given by:

$$
\lambda_{j}=\lambda_{j-4}(j=5,6)
$$

and the coefficients $B_{j}(j=1,2,3,4,5,6)$ are given by:

$$
B_{j}=-\frac{\lambda_{j}^{2}+\beta_{2}}{\beta_{1} \lambda_{j}}
$$

$\omega_{i}(i=1, \ldots, 6)$ are given in Appendix (A1).

Let's consider the jumps of displacements across the crack lips $y=0$ denoted by $\langle\bar{u}\rangle$ and $\langle\bar{v}\rangle$ :

$$
\left\{\begin{array}{l}
\langle\bar{u}\rangle=\bar{u}\left(X, 0^{+}\right)-\bar{u}\left(X, 0^{-}\right) \\
\langle\bar{v}\rangle=\bar{v}\left(X, 0^{+}\right)-\bar{v}\left(X, 0^{-}\right)
\end{array}\right.
$$


The dislocation density functions $\bar{f}_{i}(X)(i=1,2)$ along the crack line can be written as follows:

$$
\left\{\begin{array}{l}
\bar{f}_{1}(X)=\frac{\partial\langle\bar{u}\rangle}{\partial X} \\
\bar{f}_{2}(X)=\frac{\partial\langle\bar{v}\rangle}{\partial X}
\end{array}\right.
$$

The functions $\bar{f}_{i}(X)(i=1,2)$ also satisfy the single-value equations:

$$
\left\{\begin{array}{l}
\int_{-1}^{1} \overline{f_{i}}(X) d X=0(i=1,2) \\
\overline{f_{i}}(X)=0(i=1,2) \quad|X| \geq 1
\end{array}\right.
$$

Physically the second equation of (44) means that the displacements are single-valued for the uncracked portion along $y=0$. By applying the Laplace and Fourier transforms to (43), we obtain:

$$
\left\{\begin{array}{l}
\tilde{\tilde{f}}_{1}(\xi)=-\mathrm{i} \xi\left[\tilde{\bar{u}}\left(0^{+}\right)-\tilde{\bar{u}}\left(0^{-}\right)\right] \\
\tilde{\tilde{f}}_{2}(\xi)=-\mathrm{i} \xi\left[\tilde{\bar{v}}\left(0^{+}\right)-\tilde{\bar{v}}\left(0^{-}\right)\right]
\end{array}\right.
$$

Where by definition and from(44), $\tilde{\tilde{f}}_{1}$ and $\tilde{\tilde{f}}_{2}$ are given by:

$$
\tilde{\tilde{f}}_{i}=\int_{0}^{\infty}\left(\int_{-1}^{1} f_{i} \cdot \mathrm{e}^{\mathrm{i} \xi X} d X\right) \cdot \mathrm{e}^{-p \tau} d \tau \quad i=1,2
$$

By applying the boundary conditions $(a)-(d)$ in (34) and (45), $C_{i}$ can be expressed as

$$
C_{i}=\mathbf{A}_{i, j}^{-1} \boldsymbol{\gamma}_{j}+\frac{1}{\mathrm{i} \xi} \mathbf{A}_{i, 5}^{-1} \tilde{\bar{f}}_{1}+\frac{1}{\mathrm{i} \xi} \mathbf{A}_{i, 6}^{-1} \tilde{\tilde{f}}_{2} \quad i=1,2, \ldots, 6
$$

Where the matrix $\mathbf{A}_{i, j}$ and $\boldsymbol{\gamma}_{j}$ are given in Appendix (A2) and (A3).

Substitute (37) into the boundary conditions (e) and (f) in (34), and applying the relationship (35), it can be shown that $f_{i}(x)(i=1,2)$ satisfy the following equations:

$$
\left\{\begin{array}{l}
\int_{-1}^{1} \bar{f}_{1}(\eta) K_{1,1}(X, \eta) d \eta+\int_{-1}^{1} \bar{f}_{2}(\eta) K_{1,2}(X, \eta) d \eta=W_{1} \\
\int_{-1}^{1} \bar{f}_{1}(\eta) K_{2,1}(X, \eta) d \eta+\int_{-1}^{1} \bar{f}_{2}(\eta) K_{2,2}(X, \eta) d \eta=W_{2}
\end{array}\right.
$$

Where $K_{i, j}$ and $W_{i}(i=1,2 ; j=1,2)$ are given in Appendix (A5) and (A6).

The solution of the integral equations of $f_{i}(x)(i=1,2)$ can be expressed in the following form:

$$
\bar{f}_{i}(X)=\frac{\overline{F_{i}}(X)}{\sqrt{1-X^{2}}}(i=1,2) .
$$

Using the Lobatto-Chebyshev method $[9,34,35]$, we can reduce the integral equations to the following algebraic equations:

$$
\left\{\begin{array}{l}
\sum_{i=1}^{n} A_{i} K_{1,1}\left(X_{j}, \eta_{i}\right) \bar{F}_{1}\left(\eta_{i}\right)+\sum_{i=1}^{n} A_{i} K_{1,2}\left(X_{j}, \eta_{i}\right) \bar{F}_{2}\left(\eta_{i}\right)=W_{1}\left(x_{j}\right) \\
\sum_{i=1}^{n} A_{i} K_{2,1}\left(X_{j}, \eta_{i}\right) \bar{F}_{1}\left(\eta_{i}\right)+\sum_{i=1}^{n} A_{i} K_{2,2}\left(X_{j}, \eta_{i}\right) \bar{F}_{2}\left(\eta_{i}\right)=W_{2}\left(x_{j}\right) \\
\sum_{i=1}^{n} A_{i} \bar{F}_{1}\left(\eta_{i}\right)=0 \\
\sum_{i=1}^{n} A_{i} \bar{F}_{2}\left(\eta_{i}\right)=0
\end{array}\right.
$$


where

$$
\left\{\begin{array}{l}
\eta_{i}=\cos \frac{(i-1) \pi}{n-1}, i=1,2, \ldots, n \\
X_{j}=\cos \frac{(2 j-1) \pi}{2(n-1)}, j=1,2, \ldots, n-1 \\
A_{1}=A_{n}=\frac{\pi}{2(n-1)} ; \\
A_{i}=\frac{\pi}{n-1}, i=2,3, \ldots, n-1 .
\end{array}\right.
$$

The functions $F_{1}$ and $F_{2}$ at the crack line can be given by resolving $\bar{F}_{1}$ and $\bar{F}_{2}$ from (50) and performing the inverse Laplace transform. The dynamic stress intensity factors $K_{\mathrm{I}}(p)$ and $K_{\mathrm{II}}(p)$ are defined as $[19,36]$

$$
\left\{\begin{array}{l}
K_{\mathrm{I}}(t)=L^{-1}\left[-\frac{1-v^{2}}{8} \sqrt{\pi} \bar{F}_{2}(1, p)\right] \\
K_{\mathrm{II}}(t)=L^{-1}\left[-\frac{1+v}{4} \sqrt{\pi} \bar{F}_{1}(1, p)\right]
\end{array}\right.
$$

where the operator $L^{-1}[$ ] denotes the inverse Laplace transform.

\section{Result and discussions}

In this work, the Poisson's ratio is set to be 0.3 for all computations. This value is close to those of most of the brittle or quasi-brittle materials used in engineering applications. The temperature fields on the crack faces and the crack extended line when the non-Fourier effect is taken into account have been studied by Chen et al. [20]. Identical results for different values of non-Fourier parameter $\tau_{1}$ have been obtained in our work and will not be presented in this paper.

The coupled effect of the non-Fourier heat conduction and the inertia forces provides complex stress fields near the crack tips, which depend additionally on the crack position with respect to the boundary of the half-plane. The influence of all these factors on the principal crack stress concentration characterized mainly the stress intensity factors (SIFs) and the crack opening distances (CODs) will be evaluated and discussed in the following sections.

\subsection{Influence of $\tau_{1}$ and $\tau_{2}$ on the stress intensity factors}

The dynamic stress intensity factors $K_{\mathrm{I}}$ and $K_{\mathrm{II}}$ can be evaluated from (52) once we obtain the solutions of the algebraic equations (50) and perform the numerical inverse of Laplace transform. Their normalized values $\left(K_{\mathrm{I}}^{*}, K_{\mathrm{II}}^{*}\right)=\frac{\left(K_{\mathrm{I}}, K_{\mathrm{II}}\right)(1-v)}{E \alpha\left(T_{\infty}-T_{0}\right) \sqrt{r}}$ are presented in the figures.

In the case when the inertia effect is not taken into account, e.g. $\tau_{2}=0$, the time evolution of the stress intensity factors are shown in Fig. 2. In this case, the results degenerate to those of the classical quasi-static thermal stress problem under non-Fourier thermal conduction condition or under conventional Fourier thermal conduction condition when $\tau_{1}=0$. We remark that our calculations recover the existing results found in the literature [20].

When the inertia effect is considered, the dynamic stress wave will interact with the non-Fourier heat conduction. In order to present more clearly the obtained results, we will firstly assess their influence separately.

Let us first neglect the non-Fourier effect by fixing $\tau_{1}=0$. The influence of the thermal inertia factor $\tau_{2}$ on the normalized dynamic SIFs when $l=1$ is depicted in Fig. 3. From this figure, we can clearly observe the influence of this parameter on the dynamic SIFs: First, we can see that the impact of $\tau_{2}$ is much stronger on $K_{\mathrm{I}}^{*}$ than on $K_{\mathrm{II}}^{*}$ in the range of $\tau_{2} \in[0,015]$. Second, as expected, the amplitudes of the SIFs increase as $\tau_{2}$ increases. Moreover, similar to the case when $\tau_{1}>0$, the time evolution of the SIFs presents an oscillating feature when $\tau_{2}$ is large.

It is to notice that the dynamic SIF $K_{\mathrm{I}}^{*}$ can be positive or negative when $\tau_{2}>0$. This anomaly comes from the fact that the crack lip contact under compression is not taken into account in this study. Consequently, the time variation of $K_{I}^{*}$ presented in Fig. 3 is only approximate. As in general, the values of $\tau_{2}$ are quite small, the errors introduced by this fact are not very important.

In practice, the peak values of the dynamic SIFs during their time evaluation are most significant in predicting the crack growth. The peak values of $K_{\mathrm{I}}^{*}$ evaluated for different $\tau_{1}$ and $\tau_{2}$ when $l=1$ are plotted in Fig. 4. Figure 4a shows the peak values of $K_{\mathrm{I}}^{*}$ when $T_{\infty}-T_{0}>0$ (heating); whereas Fig. $4 \mathrm{~b}$ shows its peak values when $T_{\infty}-T_{0}<0$ (cooling). We notice 

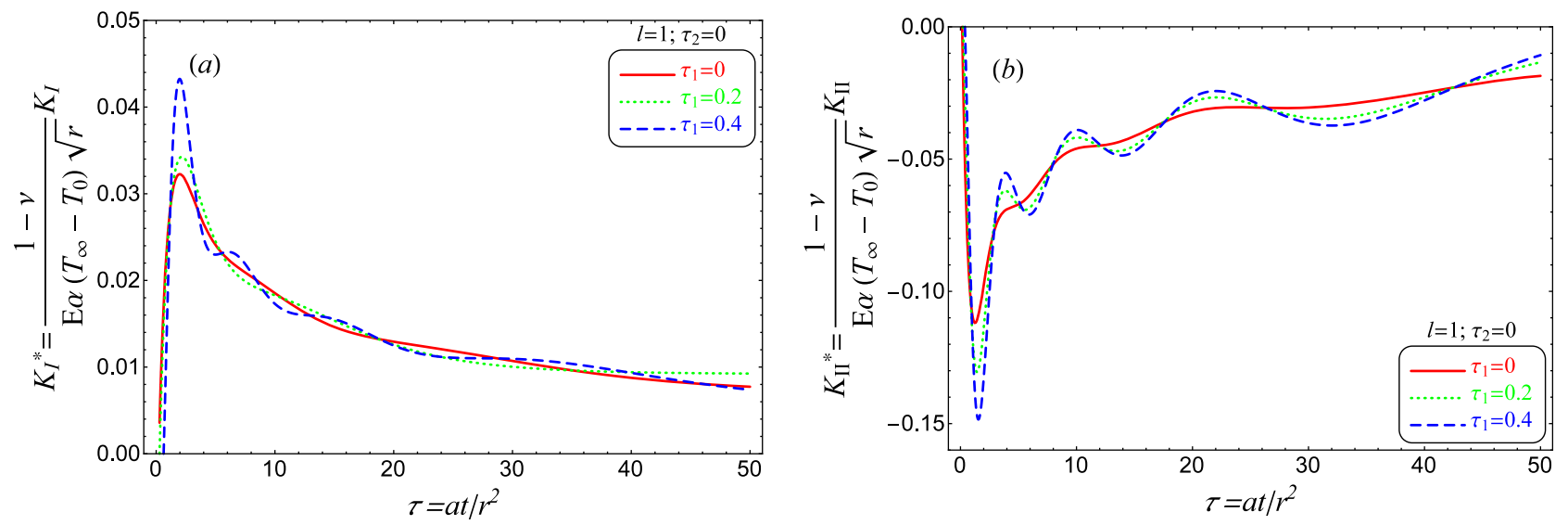

Fig. 2 Normalized stress intensity factors $(a)$ for mode-I SIFs $(b)$ for mode-II SIFs versus time for different $\tau_{1}$ when $l$ $=1$ and $\tau_{2}=0$.
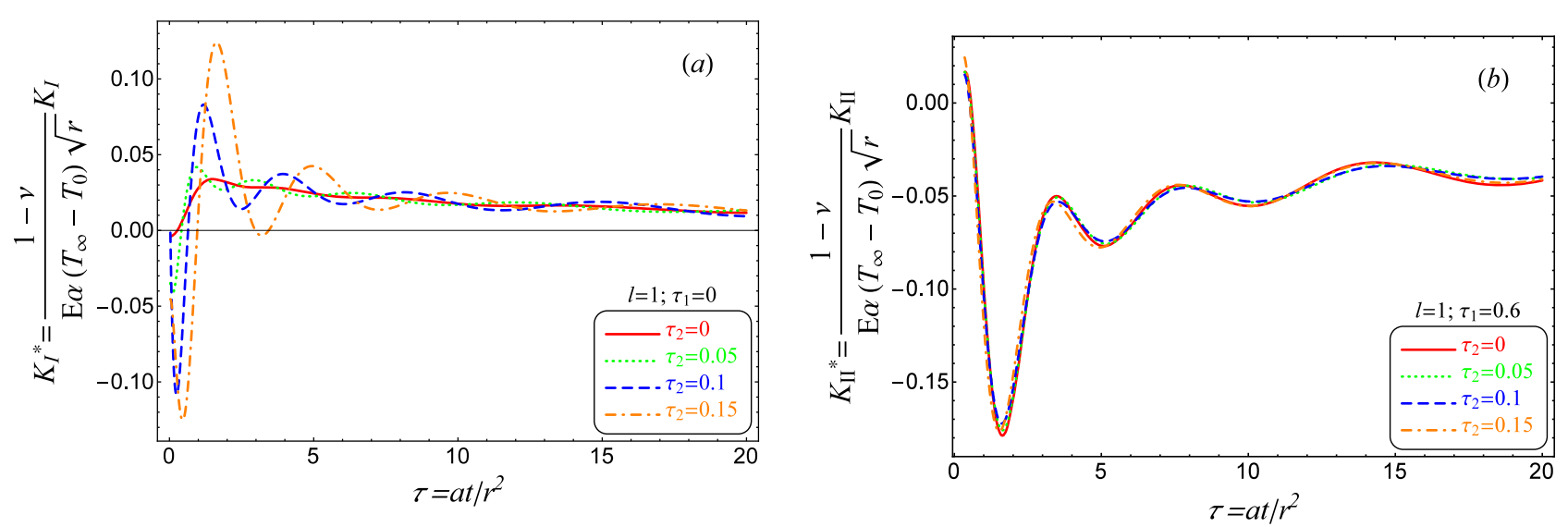

Fig. 3 Normalized stress intensity factors $(a)$ for mode-I SIFs $(b)$ for mode-II SIFs versus time for different $\tau_{2}$ when $l$ $=1$ and $\tau_{1}=0$.
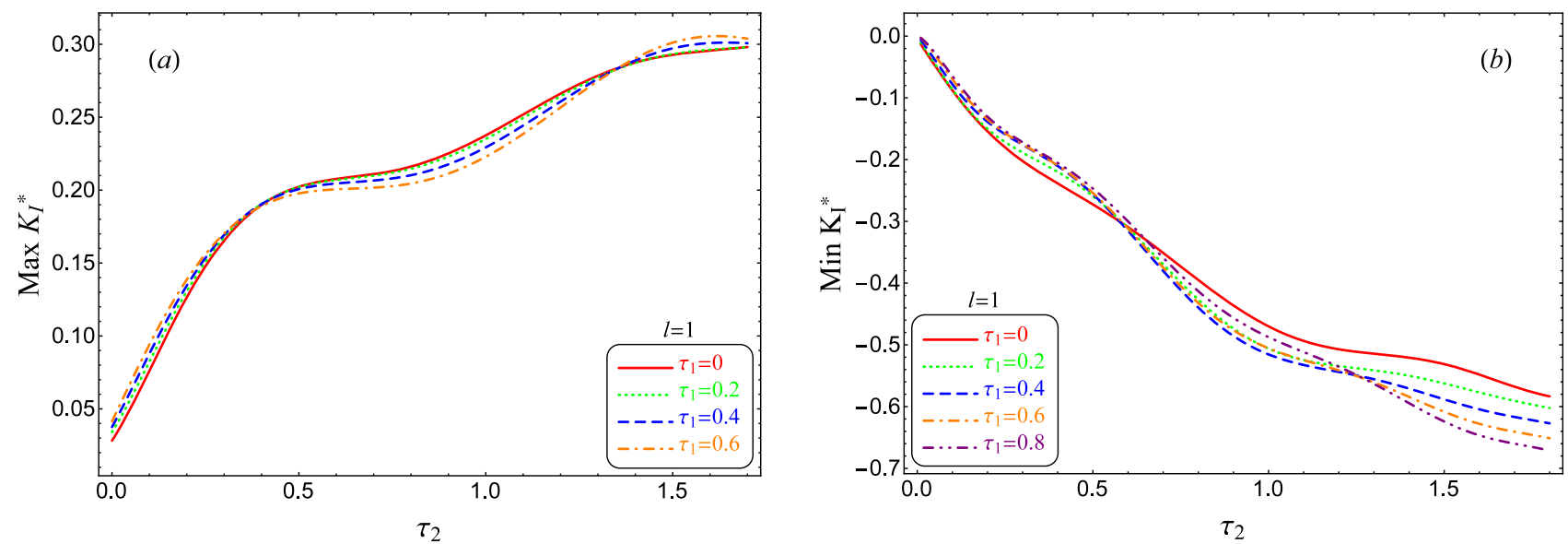

Fig. 4 The variation of peak-values of $K_{\mathrm{I}}^{*}$ versus $\tau_{2}$ for different $\tau_{1}$ for $l=1$ (a) when $T_{\infty}-T_{0}>0$ (heating) and (b) when $T_{\infty}-T_{0}<0$ (cooling).

that Fig. $4 \mathrm{~b}$ is exact while Fig. 4a is approximate due to the surface contact of the crack. We can observe that the maximum values of $K_{\mathrm{I}}^{*}$ can be increased significantly by increasing the inertia factor $\tau_{2}$, whereas the non-Fourier factor $\tau_{1}$ has a far smaller impact on $K_{\mathrm{I}}^{*}$ than $\tau_{2}$.

In Fig. 5, the peak values of $K_{\mathrm{II}}^{*}$ are illustrated as function of $\tau_{1}$ and $\tau_{2}$. It appears that the responses of $K_{\mathrm{I}}^{*}$ and $K_{\mathrm{II}}^{*}$ to these two parameters are quite different. Globally speaking, the impact of $\tau_{1}$ and $\tau_{2}$ has rather a similar order of magnitude 


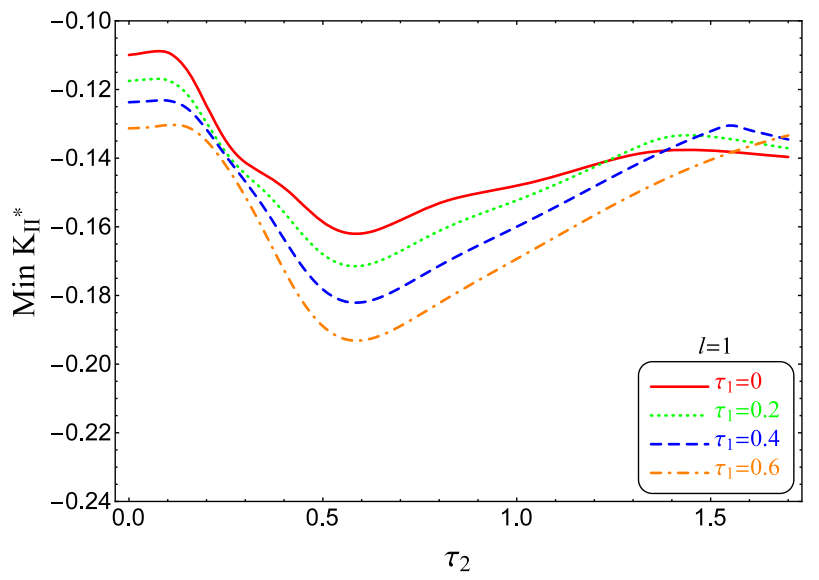

Fig. 5 the variation of peak-values of $K_{\mathrm{II}}^{*}$ versus $\tau_{2}$ for different $\tau_{1}$ for $l=1$.

for the mode-II SIF. At small $\tau_{2}$, the parameter $\tau_{1}$ is predominant, after that the peak values of $K_{\mathrm{II}}^{*}$ increase rapidly when $\tau_{2}$ increases, and attain maximums at about $\tau_{2} \cong 0.55$ before falling down afterward.

\subsection{Influence of $\tau_{1}$ and $\tau_{2}$ on the crack displacement jumps}

Apart from the dynamic SIFs, the crack opening distances (CODs) are also important parameters for predicting crack growth. The CODs can be defined in different manners. Whatever the definition, they can be calculated when knowing the displacement jumps between the crack-lips, which can be calculated by integrating the dislocation density function found from Eq. (50), then by performing the Laplace inverse transforms.

As above, we first study separately the influence of the parameters $\tau_{1}$ and $\tau_{2}$ on the displacement jumps.

Figure 6 illustrates both the mode-I and mode-II maximal displacement jumps for different $\tau_{1}$ values by fixing $\tau_{2}=0$ and $l=1 . \tau$ in the legends stand for the dimensionless time when the jumps of displacement reach the maximum value, which has the same meaning in Fig. 7 and Fig. 10. The times at which the maximal displacement jumps are reached are also indicated. This figure shows that the displacement jumps increase as $\tau_{1}$ increases. Moreover, it appears that its impact on mode-I COD is more important than on that of mode-II.
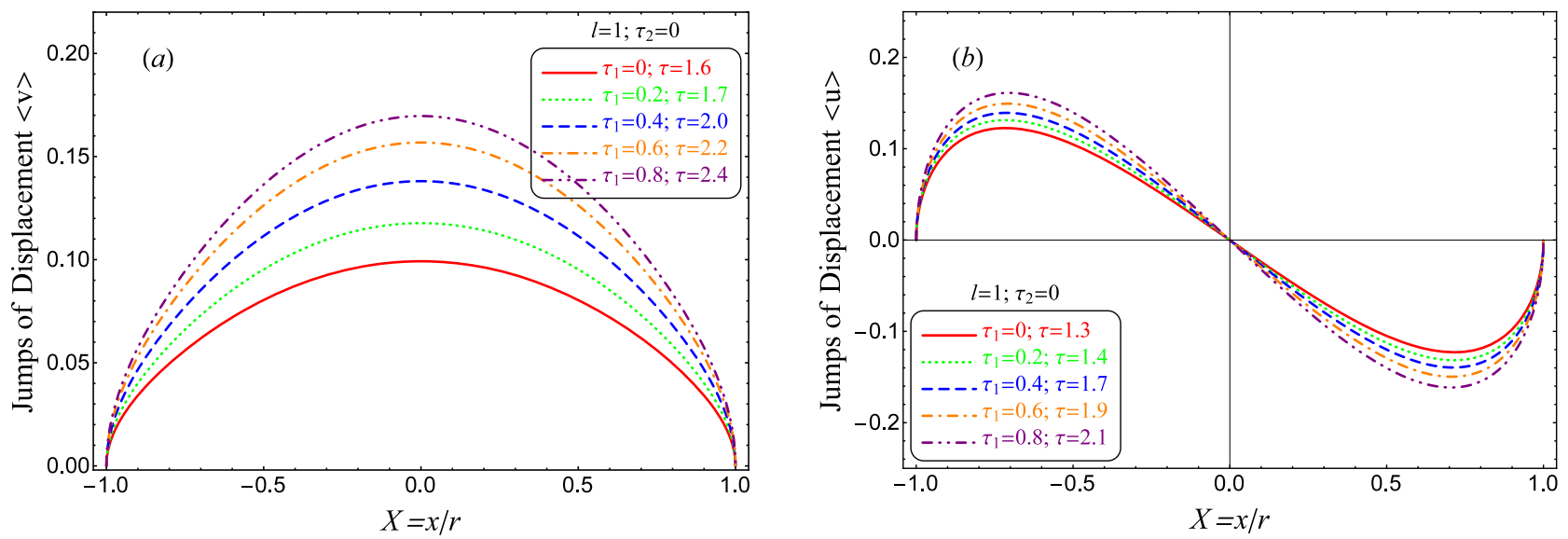

Fig. 6 Normalized displacement jumps $\Delta v$ and $\Delta u$ of the crack lips for different $\tau_{1}$ when $\tau_{2}=0$.

Similarly, we show in Fig. 7 the influence of $\tau_{2}$ on the crack displacement jump by fixing $\tau_{1}=0$ and $l=1$. Clearly, the increase of the inertia factor leads to more important in mode I crack opening. However, in mode II crack opening, the differences of displacement $<u>$ increase slowly with $\tau_{2}$.

Figure 8 shows the peak values of the maximal displacement jumps between the crack lips versus $\tau_{2}$ for different $\tau_{1}$. It is worthy to notice that the mode-I maximal displacement jumps occur always at the midpoint of the crack, whereas the mode-II maximal displacement jumps are located at the two sides of the midpoint. We can observe a clear similarity between Fig. 7 and Fig. 4 in which same diagram is drawn for the dynamic SIFs. The displacement jump $<u>$ becomes larger with the increase of $\tau_{1}$. It increases monotonically with $\tau_{2}$ at the interval $0<\tau_{2}<0.6$ and decrease monotonically 

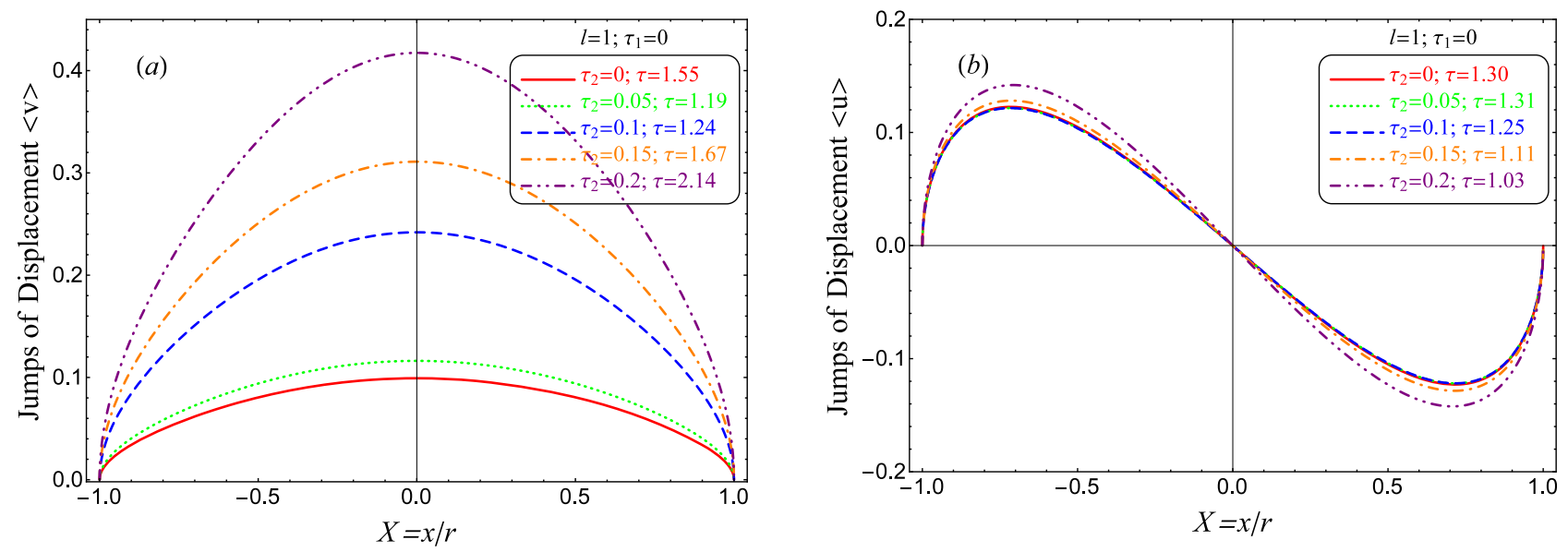

Fig. 7 Normalized displacement jumps $\Delta v$ and $\Delta u$ of the crack lips for different $\tau_{2}$ when $\tau_{1}=0$.
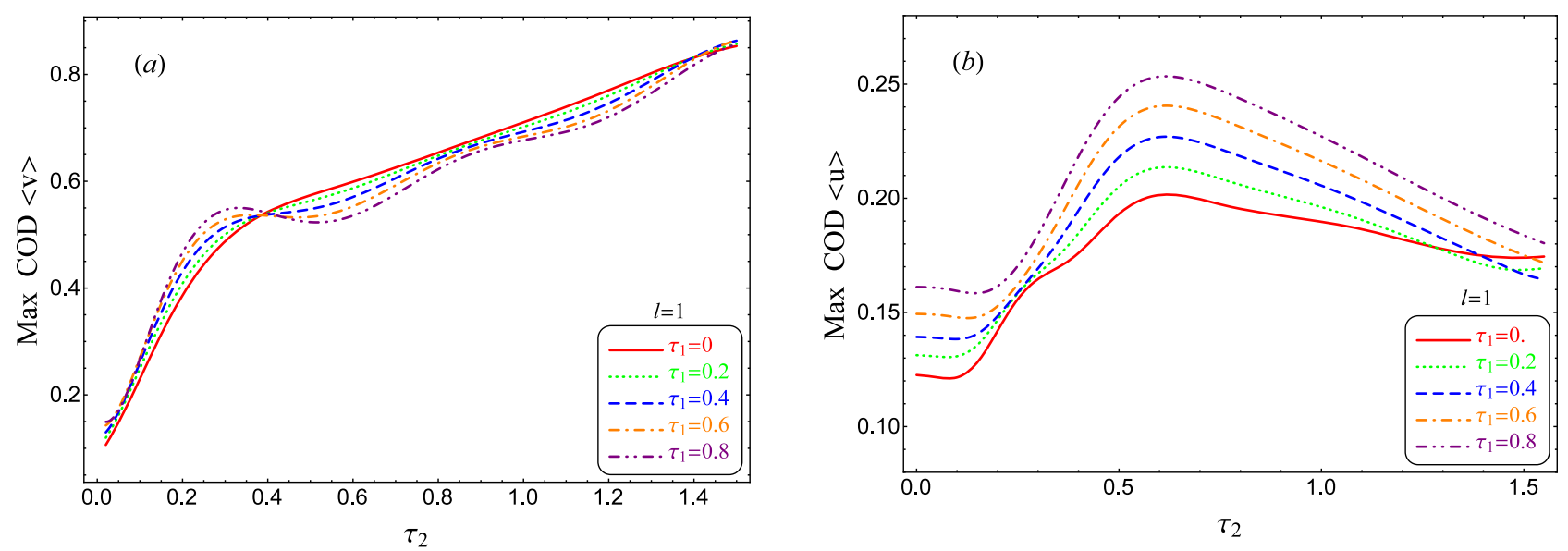

Fig. 8 Peak values of the normalized displacement jumps versus $\tau_{2}$ for different $\tau_{1}$.

with $\tau_{2}$ at the interval $0.6<\tau_{2}<1.5$. The displacement jump $<v>$ becomes larger with the increase of $\tau_{2}$. However, this increasing curve becomes slightly oscillating when $\tau_{1}$ increases.

\subsection{Influence of $l$}

It is clear that the stress field near the crack is influenced by the distance between the crack and the boundary characterized by the parameter $l$. From the engineering point of view, it is interesting to know the most unfavorable value $l$ for which the crack stress concentration is the most serious. Figures 9 and 10 show the influence of this parameter on the stress intensity factor and the crack openings. In these simulations, the inertia factor $\tau_{2}$ is taken to be 0.2 afterward.

The variation of maximal normalized SIFs $K_{\mathrm{I}}^{*}$ and $K_{\mathrm{II}}^{*}$ versus $l$ are shown in Fig. 9 for different $\tau_{1}$ when $\tau_{2}=0.2$. The starting abscissa of the curves is $l=0.1$ instead of 0 . It is clear that the absolute values of the SIFs are not simple monotonically increasing or monotonically decreasing with $l$. We can remark that their maximal values occur at $l \cong 1$ for both mode-I and mode-II curves for almost all values of $\tau_{1}$ considered.

Figure 10 shows the maximum displacement jumps of between crack lips versus the dimensionless coordinate for different $l$ when $\tau_{1}=0$ and $\tau_{2}=0.2$. It appears that the mode-I displacement jump decreases as $l$ increases. However, this tendency is no longer true in the mode-II case. To observe better the influence of $l$ on CODs, we plot in Figure 11 the maximum of the crack displacement jumps versus $l$ for different $\tau_{1}$ under the condition $\tau_{2}=0.2$. We can observe that the mode-I displacement jumps first increase and then decrease as $l$ increase. The maximal displacement jumps for different values of $\tau_{1}$ are found at points between $0.2<l<1$. For the mode-II case, overall, the maximum displacement jumps decrease as the $l$ increases whereas small oscillations can appear with the increase of $\tau_{1}$. Both the mode-I and mode-II displacement jumps monotonically decrease with $l$ when $l>1$. This result agrees well with that found in the literature [20]. 

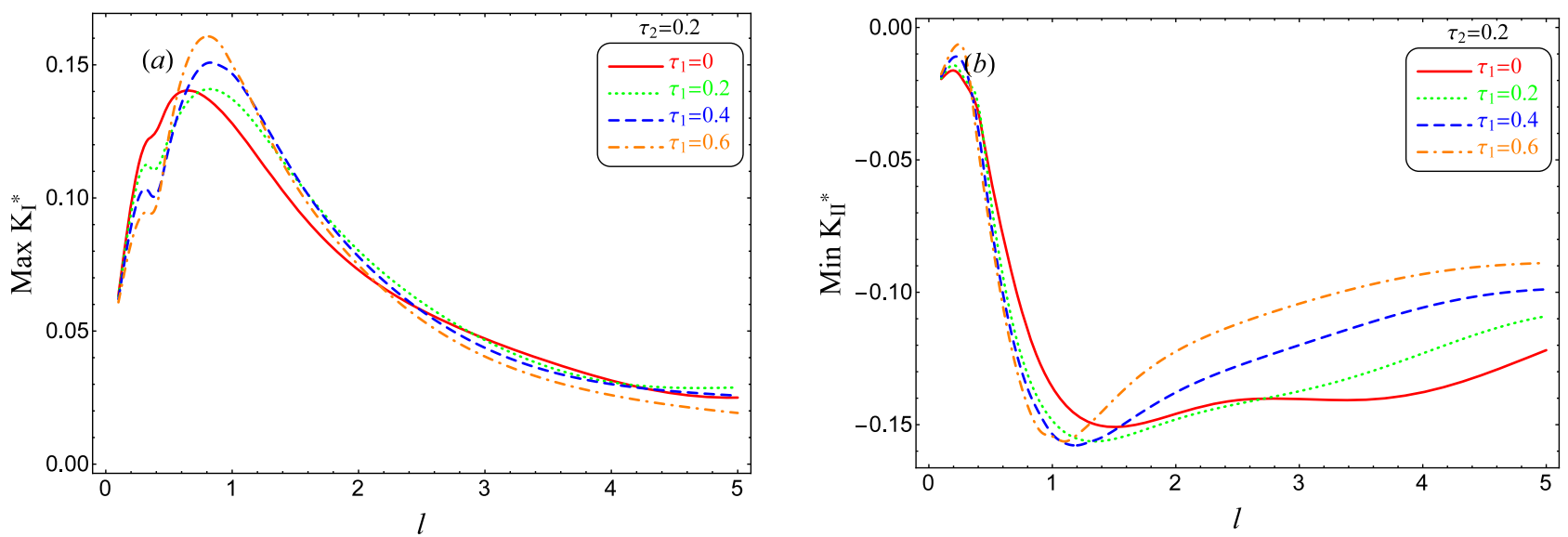

Fig. 9 Variation of maximum $K_{\mathrm{I}}{ }^{*}$ and $K_{\mathrm{II}}{ }^{*}$ versus $l$ for different $\tau_{1}$ when $\tau_{2}=0.2$.
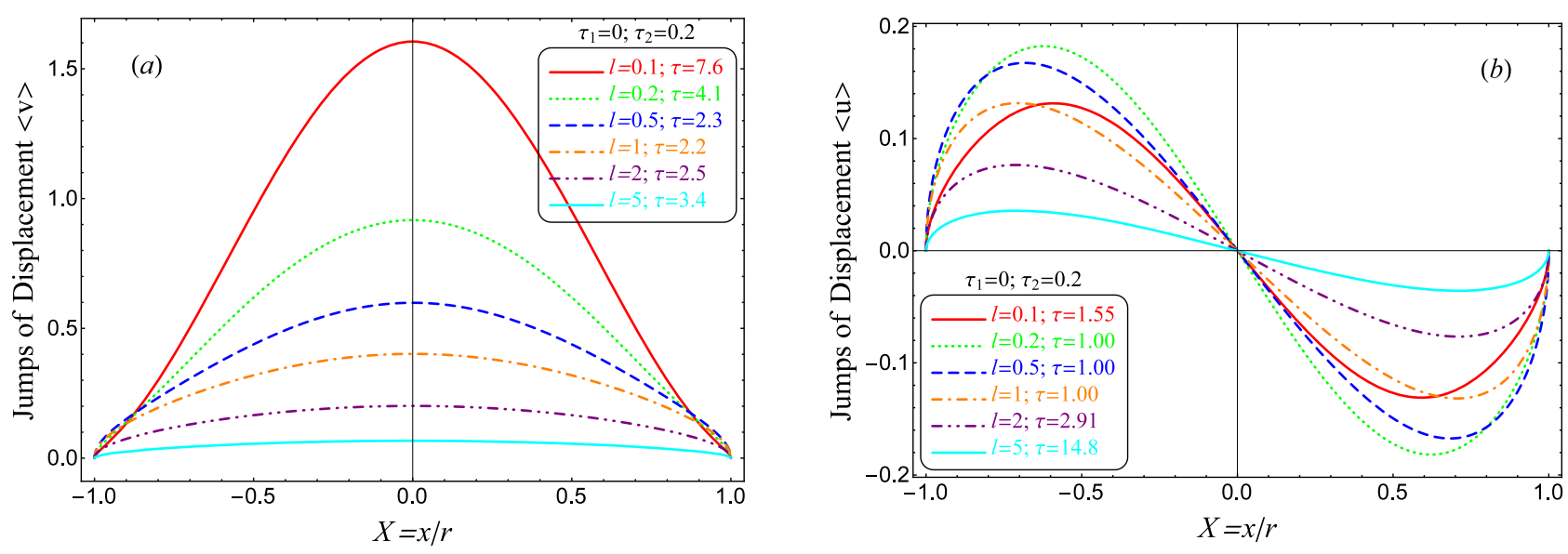

Fig. 10 Maximum displacement jumps $\Delta v$ and $\Delta u$ for different $l$ and $\tau_{1}$ when $\tau_{2}=0.2$.
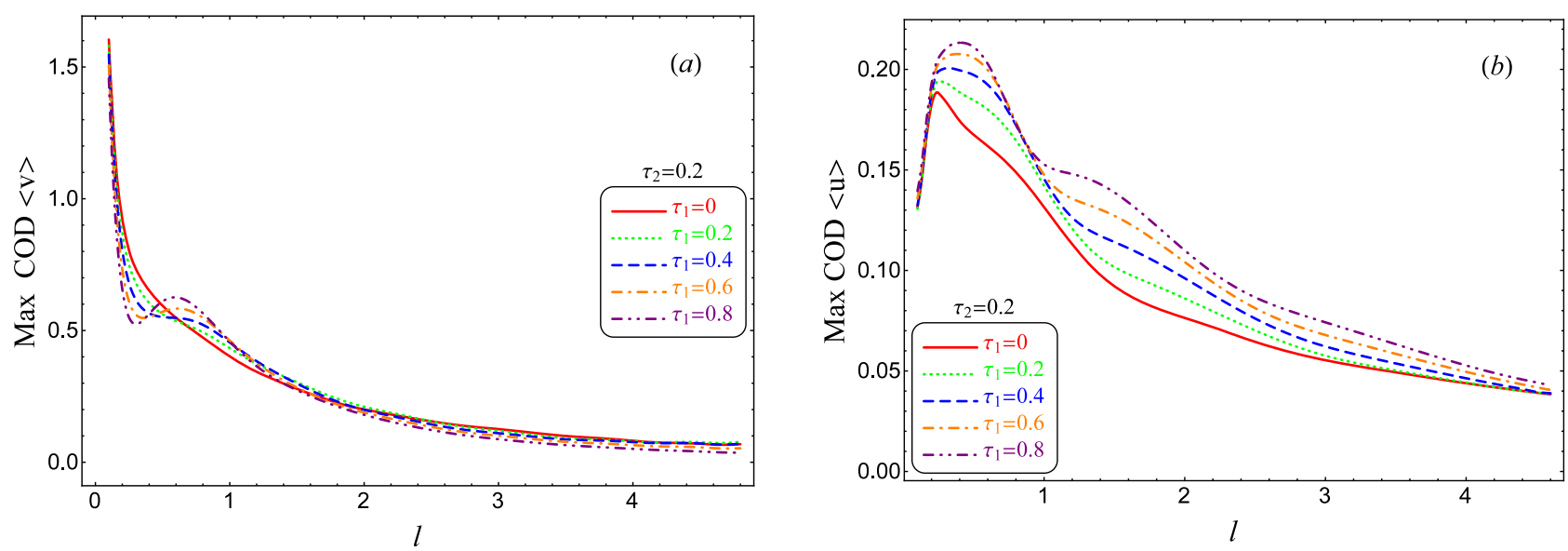

Fig. 11 Variation of maximal normalized displacement jumps $\Delta v$ and $\Delta u$ versus $l$ for different $\tau_{1}$ when $\tau_{2}=0.2$. $\Delta v$ is measured at the midpoints of the crack; $\Delta u$ is measured at the points of the crack where $\Delta u$ is maximal.

\section{Conclusions}

In this paper, the inertia force and the non-Fourier heat conduction condition are considered to investigate the transient thermal stress problem of an insulated crack parallel to the boundary of a thermal-elastic half-plane under temperature impact loading. The current mixed boundary value problem is formulated in terms of Laplace and Fourier transforms techniques and the established singular integral equations are solved using numerical methods. Temperature and stress fields in the time domain are obtained by using an asymptotic analysis and inverse Laplace transform. The dynamic stress 
intensity factors and the displacement jumps across the crack surfaces are evaluated for different values of parameters $\tau_{1}$, $\tau_{2}$, and $l$, characterizing the non-Fourier effect, the inertia effect and the distance between the crack and the half-plane boundary, respectively.

Generally speaking, the amplitude of the stress intensity factors and the crack displacement jump increases as $\tau_{1}$ and $\tau_{2}$ increase. Nevertheless, we can see that the values of mode II SIF are relatively insensitive to $\tau_{1}$ and $\tau_{2}$ whereas the mode I SIF is dramatically affected by $\tau_{2}$. The results found are useful for better understanding of the thermo-mechanical behaviors of advanced materials under a thermal shock loading. Moreover, they also constitute an exact solution of the problem and can be used to assess the accuracy of the numerical analyses.

In this preliminary work we considered a semi-infinite homogeneous cracked body. The method developed also permits us to study crack behaviors in layered body with finite dimensions in which the non-Fourier effect and inertia effect are much more pronounced, especially in the case of small-size or small-time scales [25]. Apart from a few rapports in the literature $[2,27,28]$, experimental results on these non-classical effects are still rare. All these aspects can be investigation directions in future works.

\section{Appendix}

$$
\left\{\begin{array}{l}
\omega_{1}=\frac{\mathrm{e}^{-2 m l}\left[p \tilde{\bar{\phi}}-2 \mathrm{ie}^{m l} \delta(\xi) \xi\right]}{2 p\left(-m^{2}+\xi^{2}+p^{2} \tau_{2}^{2}\right)} \\
\omega_{2}=\frac{\tilde{\bar{\phi}}}{2\left(m^{2}-\xi^{2}-p^{2} \tau_{2}^{2}\right)} \\
\omega_{3}=\frac{\mathrm{e}^{-2 m l} m\left[\mathrm{ip} \tilde{\bar{\phi}}+2 \mathrm{e}^{m l} \delta(\xi) \xi\right]}{2 p \xi\left(-m^{2}+\xi^{2}+p^{2} \tau_{2}^{2}\right)} \\
\omega_{4}=\frac{\mathrm{i} \tilde{\bar{\phi}} m}{2 \xi\left(-m^{2}+\xi^{2}+p^{2} \tau_{2}^{2}\right)} \\
\omega_{5}=-\frac{\left(1+\mathrm{e}^{-2 m l}\right) p \tilde{\bar{\phi}}-2 \mathrm{ie}^{-m l} \delta(\xi) \xi}{2 p\left(m^{2}-\xi^{2}-p^{2} \tau_{2}^{2}\right)} \\
\omega_{6}=\frac{\mathrm{ie}^{-2 m l} m\left[\left(1+\mathrm{e}^{2 m l}\right) p \tilde{\bar{\phi}}-2 \mathrm{ie}^{m l} \delta(\xi) \xi\right]}{2 p \xi\left(-m^{2}+\xi^{2}+p^{2} \tau_{2}^{2}\right)}
\end{array}\right.
$$

where $\delta(\xi)=\left\{\begin{array}{ll}\infty, & \xi=0 \\ 0, & \xi \neq 0\end{array}\right.$.

$$
\left\{\begin{array}{l}
\mathbf{A}_{1, j}= \begin{cases}\left(\mathrm{i} \xi \nu+b_{j} \lambda_{j}\right) \mathrm{e}^{-\lambda_{j} l} & j=1,2,3,4 \\
0 & j=5,6\end{cases} \\
\mathbf{A}_{2, j}= \begin{cases}\left(\mathrm{i} \xi b_{j}+\lambda_{j}\right) \mathrm{e}^{-\lambda_{j} l} & j=1,2,3,4 \\
0 & j=5,6\end{cases} \\
\mathbf{A}_{3, j}= \begin{cases}\mathrm{i} \xi \nu+b_{j} \lambda_{j} & j=1,2,3,4 \\
0 & j=5,6\end{cases} \\
\mathbf{A}_{4, j}= \begin{cases}\mathrm{i} \xi b_{j}+\lambda_{j} & j=1,2,3,4 \\
0 & j=5,6\end{cases} \\
\mathbf{A}_{5, j}= \begin{cases}-1 & j=1,2,3,4 \\
1 & j=5,6\end{cases} \\
\mathbf{A}_{6, j}= \begin{cases}-b_{i} & j=1,2,3,4 \\
b_{i} & j=5,6\end{cases}
\end{array}\right.
$$




$$
\begin{aligned}
& \left\{\begin{array}{l}
\boldsymbol{\gamma}_{1}=\frac{(v-1) \xi^{2}-p^{2} \tau_{2}^{2}}{p\left(p+p^{2} \tau_{1}-p^{2} \tau_{2}^{2}\right)} \delta(\xi) \\
\boldsymbol{\gamma}_{2}=-\frac{2 m p \mathrm{e}^{-m l}}{p\left(m^{2}-\xi^{2}-p^{2} \tau_{2}^{2}\right)} \tilde{\bar{\phi}}+\frac{2 \mathrm{i} m \xi}{p\left(m^{2}-\xi^{2}-p^{2} \tau_{2}^{2}\right)} \delta(\xi) \\
\boldsymbol{\gamma}_{3}=\frac{\mathrm{i}\left((1-v) \xi^{2}-p^{2} \tau_{2}^{2}\right)}{\xi\left(p+p^{2} \tau_{1}-p^{2} \tau_{2}^{2}\right)} \tilde{\bar{\phi}} \\
\boldsymbol{\gamma}_{4}=0 \\
\boldsymbol{\gamma}_{5}=\frac{1}{p+p^{2} \tau_{1}-p^{2} \tau_{2}^{2}} \tilde{\bar{\phi}} \\
\boldsymbol{\gamma}_{6}=0
\end{array}\right. \\
& \left\{\begin{array}{l}
K_{1,1}=2 \int_{0}^{\infty} \sum_{i=5}^{6}\left[\left(v+\frac{b_{i} \lambda_{i}}{v \xi}\right) \mathbf{A}_{i, 5}^{-1}\right] \cos (\xi(X-\eta)) d \xi \\
K_{1,2}=2 \int_{0}^{\infty}\left(-\mathrm{i} \sum_{i=5}^{6}\left[\left(v+\frac{b_{i} \lambda_{i}}{\nu \xi}\right) \mathbf{A}_{i, 6}^{-1}\right]-\frac{1-v^{2}}{4}\right) \sin (\xi(X-\eta)) d \xi+\frac{1-v^{2}}{2} \frac{1}{X-\eta} \\
W_{1}=\int_{-1}^{1} \bar{\phi}(\eta) w_{1}(X-\eta) d \eta+\frac{\mathrm{e}^{-\tau_{2} p l}-\mathrm{e}^{-\sqrt{p\left(1+\tau_{1} p\right)} l}}{1+\tau_{1} p-\tau_{2}^{2} p} \tau_{2}^{2} \\
K_{2,1}=2 \int_{0}^{\infty}\left(-\mathrm{i} \sum_{i=5}^{6}\left[\left(\frac{\lambda_{i}}{\mathrm{i} \xi}+b_{i}\right) \mathbf{A}_{i, 5}^{-1}\right]-\frac{1-v}{2}\right) \sin (\xi(X-\eta)) d \xi+\frac{1-v}{X-\eta} \\
K_{2,2}=2 \int_{0}^{\infty} \sum_{i=5}^{6}\left[\left(\frac{\lambda_{i}}{\mathrm{i} \xi}+b_{i}\right) \mathbf{A}_{i, 6}^{-1}\right] \cos (\xi(X-\eta)) d \xi \\
W_{2}=\int_{-1}^{1} \bar{\phi}(\eta) w_{2}(X-\eta) d \eta
\end{array}\right.
\end{aligned}
$$

where

$$
\left\{\begin{array}{l}
w_{1}=\int_{0}^{\infty}\left[\sum_{i=5}^{6}\left(\xi \nu-\mathrm{i} b_{i} \lambda_{i}\right) w_{0}-\frac{1}{2}\left(\xi-v \xi+\frac{p^{2} \tau_{2}^{2}}{\xi}\right)\left(1+\mathrm{e}^{-2 m l}\right)\right] \sin [\xi(X-\eta)] d \xi \\
w_{2}=\int_{0}^{\infty}\left[\sum_{i=5}^{6}\left(\lambda_{i}+\mathrm{i} \xi b_{i}\right) w_{0}-m\left(1+\mathrm{e}^{-2 m l}\right)\right] \cos [\xi(X-\eta)] d \xi
\end{array}\right.
$$

and

$$
w_{0}=\frac{2}{p\left(1+\tau_{1} p-\tau_{2}^{2} p\right)} \cdot\left[-2 m \mathrm{e}^{-m l} \mathbf{A}_{i, 2}+\mathrm{i}\left(\xi-v \xi+\frac{p^{2} \tau_{2}^{2}}{\xi}\right) \mathbf{A}_{i, 3}+\mathbf{A}_{i, 5}\right]
$$

Acknowledgement This work was supported by the funding from the French ANR program T-Shock ANR-10-INTB-0915.

\section{References}

[1] K. J. Baumeister and T. D. Hamill, Hyperbolic heat-conduction equation - A solution for the semi-infinite body problem, J. Heat Trans. 91, 543-548 (1969).

[2] H. Kogawa, M. Futakawa, S. Isikura, K. Kikuchi, R. Hino, and M. Eto, Inertia effect on thermal shock by laser beam shot, International Journal of Impact Engineering 25, 17-28 (2001).

[3] L. Hector, W.-S. Kim, and M. Özisik, Hyperbolic heat conduction due to a mode locked laser pulse train, Int. J. Eng. Sci. 30, 1731-1744 (1992).

[4] M. Babaei and Z. Chen, Hyperbolic heat conduction in a functionally graded hollow sphere, Int. J. Thermophys. 29, 1457-1469 (2008). 
[5] I. Sevostianov and M. Kachanov, Anisotropic thermal conductivities of plasma-sprayed thermal barrier coatings in relation to the microstructure, J. Therm. Spray Techn. 9, 478-482 (2000).

[6] M. Williams, The stresses around a fault or crack in dissimilar media, B. Seismol. Soc. Am. 49, 199-204 (1959).

[7] G. C. Sih, On the singular character of thermal stresses near a crack tip, J. Appl. Mech. 29, 587-589 (1962).

[8] G. D. Gupta, An integral equation approach to the semi-infinite strip problem, J. Appl. Mech. 40, 948-954 (1973).

[9] P. Theocaris and N. Ioakimidis, Numerical integration methods for the solution of singular integral equations (for crack tip stress intensity factor evaluation in elastic media), Q. Appl. Math. 35, 173-183 (1977).

[10] Z.-H. Jin and N. Noda, An internal crack parallel to the boundary of a nonhomogeneous half plane under thermal loading, Int. J. Eng. Sci. 31, 793-806 (1993).

[11] Y. Chen and F. Erdogan, The interface crack problem for a nonhomogeneous coating bonded to a homogeneous substrate, J. Mech. Phys. Solids 44, 771-787 (1996).

[12] F. Erdogan and B. Wu, Crack problems in FGM layers under thermal stresses, Journal of Thermal Stresses 19, 237-265 (1996).

[13] S. Itou, Thermal stresses around a crack in the nonhomogeneous interfacial layer between two dissimilar elastic half-planes, Int. J. Solids Struct. 41, 923-945 (2004).

[14] Y. Zhou, X. Li, and J. Qin, Transient thermal stress analysis of orthotropic functionally graded materials with a crack, Journal of Thermal Stresses 30, 1211-1231 (2007).

[15] S. Chi, Cracking in coating-substrate composites with multi-layered and FGM coatings, Eng. Fract. Mech. 70, 1227-1243 (2003).

[16] S. Dag and F. Erdogan, A surface crack in a graded medium under general loading conditions, J. Appl. Mech. 69, 580-588 (2002).

[17] R. Bagheri and M. Ayatollahi, Multiple moving cracks in a functionally graded strip, Appl. Math. Model. 36, 4677-4686 (2012).

[18] Y. F. Shao, X. H. Xu, S. H. Meng, G. H. Bai, C. P. Jiang, and F. Song, Crack patterns in ceramic plates after quenching, J. Am. Ceram. Soc. 93, 3006-3008 (2010).

[19] K. Hu and Z. Chen, Thermoelastic analysis of a partially insulated crack in a strip under thermal impact loading using the hyperbolic heat conduction theory, Int. J. Eng. Sci. 51, 144-160 (2012).

[20] Z. T. Chen and K. Q. Hu, Thermo-elastic analysis of a cracked half-plane under a thermal shock impact using the hyperbolic heat conduction theory, Journal of Thermal Stresses 35, 342-362 (2012).

[21] Z. Chen and S. Meguid, The transient response of a piezoelectric strip with a vertical crack under electromechanical impact load, Int. J. Solids Struct. 37, 6051-6062 (2000).

[22] J. Fu, Z. Chen, L. Qian, and Y. Xu, Non-Fourier thermoelastic behavior of a hollow cylinder with an embedded or edge circumferential crack, Eng. Fract. Mech. 128, 103-120 (2014).

[23] B.-L. Wang, Transient thermal cracking associated with non-classical heat conduction in cylindrical coordinate system, Acta Mech. Sinica 29, 211-218 (2013).

[24] N. Naotake, M. Yasuhiro, and N. Hiroshi, Coupled thermoplastic problem of an infinite solid containing a penny-shaped crack, Int. J. Eng. Sci. 28, 347-353 (1990).

[25] B. Boley and J. Weiner, Theory of Thermal Stresses (Dover, New York 1997).

[26] W. Kaminski, Hyperbolic heat conduction equation for materials with a nonhomogeneous inner structure, J. Heat Trans. 112, 555-560 (1990).

[27] A. Graßmann and F. Peters, Experimental investigation of heat conduction in wet sand, Heat Mass Transfer 35, 289-294 (1999).

[28] W. Roetzel, N. Putra, and S. K. Das, Experiment and analysis for non-Fourier conduction in materials with non-homogeneous inner structure, Int. J. Therm. Sci. 42, 541-552 (2003).

[29] I. Keles and C. Conker, Transient hyperbolic heat conduction in thick-walled FGM cylinders and spheres with exponentiallyvarying properties, European Journal of Mechanics-A/Solids 30, 449-455 (2011).

[30] M. K. Miller and W. T. Guy Jr., Numerical inversion of the Laplace transform by use of Jacobi polynomials, Siam J. Numer. Anal. 3, 624-635 (1966).

[31] Z. Chen, Interfacial coplanar cracks in piezoelectric bi-material systems under pure mechanical impact loading, Int. J. Solids Struct. 43, 5085-5099 (2006).

[32] K. Q. Hu and Z. T. Chen, Thermoelastic analysis of a partially insulated crack in a strip under thermal impact loading using the hyperbolic heat conduction theory, Int. J. Eng. Sci. 51, 144-160 (2012).

[33] E. Brown and F. Erdogan, Thermal stresses in bonded materials containing cuts on the interface, Int. J. Eng. Sci. 6, 517-529 (1968).

[34] F. Delale and F. Erdogan, Effect of transverse shear and material orthotropy in a cracked spherical cap, Int. J. Solids Struct. 15, 907-926 (1979).

[35] F. Erdogan, G. D. Gupta, and, T. S. Cook, Numerical Solution of Singular Integral Equations. in: Methods of Analysis and Solutions of Crack Problems (Springer, 1973). pp. 368-425.

[36] Z. T. Chen and K. Q. Hu, Hyperbolic heat conduction in a cracked thermoelastic half-plane bonded to a coating, Int. J. Thermophys. 33, 895-912 (2012). 